\title{
PsychoGrapho: A Double-Blind Cohort Study of Jungian Typology and Handwriting Analysis*
}

\author{
E. Marcus Barnes, Jan Six \\ Institute of Neuroscience and Consciousness Studies, \\ Austin, USA
}

\author{
Raymond C. Hawkins II \\ Fielding Graduate University \& University of Texas at \\ Austin, Austin, USA
}

\begin{abstract}
Previous research studies on the relationship between Jungian typology and handwriting analysis have been informal, inconclusive, or methodologically flawed. In the present double-blind cohort study, four expert handwriting analysts, who were knowledgeable about Jungian theory and the Myers-Briggs Type Indicator (MBTI), independently determined the four letter MBTI types of participants based upon blind interpretation of their handwriting samples. The results were that the handwriting analysts' accuracy of assigning four-letter MBTI types (or correctly identifying the cohort's preferences on each of the four dichotomies) was no better than chance, and the analysts' ratings did not agree with one another. We discuss the limitations of our study, and the potential next research steps.
\end{abstract}

Keywords: Jungian typology, personality traits, Myers-Briggs Type Indicator (MBTI), handwriting analysis, double-blind design

\section{Introduction}

In the multidisciplinary field of consciousness studies, the taxonomy of individual differences has always been of considerable importance. One of the more successful taxonomical models of consciousness was first developed by Carl Gustav Jung in the early 20th century (Jung, 1923/1971; 1933) as the theory of psychological types. Over the years, formal psychometric instruments have been developed based on Jungian typology, the Gray-Wheelwright Jungian Type Survey (GWJTS) (Gray, Wheelwright, \& Wheelwright, 1964) being the earliest of these. However, the later Myers-Briggs Type Indicator@ (MBTI) (Myers, 1980) has become far more widely known (Keirsey \& Bates, 1984; Center for Applications of Psychological Type (CAPT), 1985). More recent instruments include the Singer-Loomis Inventory of Personality (SLIP) (Singer, Loomis, Kirkhart, \& Kirkhart, 1996), Exploring You (Type Resources, 2009), and the Majors Personality Type Elements (PT-E) (Majors, 2012). The MBTI taxonomy of 16 personality types classified using a 4-letter personality type code has become particularly prominent in the field, to the point that Jungian personality types tend to be described by default using the 4-letter personality type code.

\footnotetext{
*Acknowledgments: We gratefully acknowledge Seth Disner, Ph.D., for his statistical consultation, and the advice of others on various phases of this project: Barry Beyerstein, John Beebe, Jamie Johnson, Tovia Katz, Robert McAlpine, Ray Moody, and Ruby Scott.

E. Marcus Barnes, BS, P.E., Institute of Neuroscience and Consciousness Studies.

Jan Six, Ph.D., Institute of Neuroscience and Consciousness Studies.

Raymond C. Hawkins II, Ph.D., ABPP, Fielding Graduate University, \& Psychology Dept., The University of Texas at Austin.
} 
Another model of individual personality differences with a long history (Nickell, 1992) is the use of "handwriting analysis" (sometimes called "graphology") to study personality types. There have been a number of efforts to formalize handwriting analysis (Lockowandt, 1976/1992; International Graphoanalysis Society (IGAS), 2010), although the theoretical underpinnings of handwriting analysis have also been challenged (Beyerstein, 1992). Clearly, it would be of great benefit to both Jungian analytic psychology and handwriting analysis - and by extension to the modeling of consciousness - if clinically sound psychometric correlations between them could be identified and validated.

Although Jungian psychologists have shown an interest in handwriting analysis for many years (Teillard, 1973), most previous attempts to cross-map Jungian type theory to psychometric outcomes of handwriting analysis have been informal, inconclusive, or methodologically flawed (Beyerstein \& Beyerstein, 1992; Lester \& Hoyd, 1981; Moore, 1982; Bayne \& O’Neill, 1988; Sevilla, 1996; Kopischke, 2001). In our study, we specifically aimed to address these challenges by using a rigorously double-blind protocol to have standardized content handwriting samples produced by a cohort of professionals with very high Jungian type certainty analyzed by a diverse group of highly experienced handwriting experts familiar with Jungian typology. To our knowledge, no previous study of Jungian typology and handwriting analysis has ever combined all of the elements italicized in the preceding sentence.

\section{Project Description and Overview}

The generic title PsychoGrapho signifies our research study because it seeks to determine the psychometric variables that may be common to both Jungian typology and handwriting analysis (graphology; graphoanalysis( $)$ ). Sponsored by the Institute for Neuroscience and Consciousness Studies (INACS), PsychoGrapho is a double-blind, cohort pilot study, whose unique design features are intended to overcome methodological weaknesses observed in various earlier investigations, as described earlier.

The main independent variable in the pilot study is the set of personality attributes inherent in Jungian analytic psychology and type theory, encapsulated in the 4-letter personality type code format. To avoid one common flaw of previous studies, Jungian personality type certainty was a major focus of the PsychoGrapho study design. Therefore, the cohort of participants who provided handwriting samples was composed exclusively of professionals with long experience in the field of Jungian psychology, who can be assumed to have an exceptionally high degree of certainty about their own 4-letter personality type code. It is worth noting that although our pilot study uses the traditional 4-letter type code format familiar from the MBTI, the MBTI was not directly administered to our cohort. In effect, PsychoGrapho uses the 4-letter type designation in a purely taxonomic sense. This approach is very similar to that used by Isabel Briggs Myers during the development of the MBTI, where she used a criterion group of friends and relatives with very high type certainty to fine-tune individual test items (Lawrence, 1986, p. 216). In the same sense, our cohort of MBTI professionals with very high type certainty can be regarded as an ideal criterion group to test against handwriting analysis outcomes. Given the theoretical and empirical durability of Jungian type theory, it can well serve as a sound touchstone against which handwriting analysis - as a psychometric instrument — can be objectively examined. To enhance the validity of the dependent variable of personality traits determined by handwriting analysis, a number of methodological precautions were taken. All handwriting samples were double blind, identifiable only by a code number uniquely matched to the participating professionals. Principal investigators and handwriting analysts remained unaware of the individual participants' 4-letter personality 
type codes. Also, the content of handwriting samples was standardized by providing a standard sample text (used by IGAS certified analysts); this was intended to avoid the representativeness cueing likely to result from variable content (Kahneman \& Tversky, 1973/1982) — a notable flaw of previous studies (e.g., Sevilla, 1996). To enhance statistical power and to dilute possible individual overconfidence biases in both MBTI professionals and handwriting analysts (Oskamp, 1965/1982), we aimed to collect samples from as large a population as feasible of both.

A secondary research objective, independent of handwriting analysis, was to investigate how the cohort group members' personal and demographic data correlate with their Jungian psychological types. These data collected using a separate questionnaire presented to cohort members at the same time as the handwriting samples will be analyzed and reported in a separate paper.

\section{Research Question}

Are the Jungian-oriented handwriting analysts able to assign a four letter MBTI type code to each of the participants' writing samples corresponding to these participants' own self-reported MBTI types? The null hypothesis is that this correspondence will not differ from chance expectations.

\section{Method}

\section{Participants and Cohort Sample}

As described in the Procedure section, the final cohort comprised 57 handwriting samples obtained from 56 individuals (24 males, 31 females, one gender unspecified), 49 of whom were European American, two Hispanic American, one African American, and one Asian American, with an average age of 57.5 years ( $S D=$ 10.3), who were confident of their MBTI four-letter type code because of their years of using, teaching, and/or writing about psychological type (mean of 21.2 years, $9.5 \mathrm{SD}$ ). The cohort's MBTI types were as follows: ISTJ (3), ISFJ (2), INFJ (5), INTJ (7), ISTP (0), ISFP (2), INFP (5), INTP (6), ESTP (0), ESFP (0), ENFP (11), $\operatorname{ENTP}(3), \operatorname{ESTJ}(1), \operatorname{ESFJ}(2), \operatorname{ENFJ}(6)$, and ENTJ (2). The four handwriting analysts comprised one male and three females, middle aged, and Caucasian. Two of their self-reported MBTI type preferences were INTJ, and the other two were INFJ, and ISTP.

\section{Procedure}

Phase I. Data Gathering and Protocol. Beginning in 2007, 57 handwriting samples were collected over a 10-month period from a selected cohort group of 56 participants. The major criterion for participant selection was confident, professional certainty of the participant regarding his or her own 4-letter personality type code, based on the participant's proficiency in Jungian analytic psychology and type theory. Each and every participant met that requirement, and adhered to a uniform, strict protocol in providing their data. Their handwriting specimens were copied from a standard text recommended by handwriting analysts. Participants also completed a demographic questionnaire querying a number of variables that could be potentially correlated with personality type. Within a 36-hour time limit, each participant returned his or her data in a sealed envelope to one of the principal investigators. To maintain a strict, double-blind protocol and participant anonymity, neither the INACS research team nor the prospective independent handwriting analysts knew any participant's personal identity or 4-letter personality type code. Handwriting specimens were identified only by a unique numerical code.

Phase II. Controlled Sample Processing and Data Entry. Phase II of the study was intended to safely process the data gathered in Phase I into a format that could be safely presented to the handwriting analysts 
without accidentally unblinding either the principal investigators or the analysts themselves. At the same time, the ancillary data, not directly related to handwriting analysis, collected in the participant questionnaires were sorted into a format that also could be safely inspected by the principal investigators without accidental unblinding. Under the supervision of one of the principal investigators, who is also a university of Texas at Austin (UT) faculty member, a UT psychology graduate student assistant was appointed as the independent person to handle the Phase II and Phase III data management, and so was unblinded to certain data categories at this stage of the project. His work included opening the sealed envelopes, sequestering the non-coded signed consent forms, and separating out and organizing the other materials. At this stage, it was found that one of the 56 participants in the cohort had copied the standardized test twice, first in a form of half-cursive printing (described by the participant as the usual form of handwriting), and then again in a cursive style. We decided to separate these two samples, which yielded 57 total handwriting samples. The coded handwriting specimens were scanned to electronic media and secluded in preparation for Phase III of the project, when they were presented to the selected handwriting analysts. Data from the coded questionnaire sheets were categorized and entered in several general data formats. The third data category, though not necessarily associated with handwriting analysis, can be the basis for additional correlational studies involving psychological type and a host of other variables, to be reported elsewhere.

Phase III. Handwriting Analysis and Jungian Typology. A pool of potential handwriting analysts who were thought also to have some expertise in Jungian psychology was assembled by both formal and informal contacts with handwriting analysts local to the Austin area or already known to one of the principal investigators, and by several extensive online searches, both by keyword and by examination of secondary sources, such as meeting reports and reference citations, by the principal scientist. Eventually 16 potential candidates were identified by these means; two more potential candidates would later be identified through referral by candidates already contacted, bringing the total initial size of the potential cohort of handwriting analysts to 18 . Online contact information was available for 12 of the initial 16 candidates, but after discussion among the research team it was decided not to contact one of them because of a potential conflict of interest. Thus, 11 potential handwriting analysts were initially contacted by email. Four of them never responded, and one of them responded but did not consider herself sufficiently qualified to participate. Thus, 7 respondents qualified as potential handwriting analysts. Although all 7 candidates were fully qualified based on their expertise in handwriting analysis and their knowledge of Jungian typology, they were encouragingly diverse in other respects. Based on their formal accreditations, both formal (IGAS, ASPG) and holistic (AHAF, IIHS, IGC) schools of handwriting analysis were well represented. Our final contingent of handwriting analysts comprised four individuals (one male and three females). Each handwriting analyst was paid an honorarium of $\$ 1,000$ for participating in this study.

The 57 handwriting specimens collected in Phase I and sorted and scanned in Phase II were presented in Phase III to this group of four expert handwriting analysts selected on the basis of their double expertise in the fields of handwriting analysis on the one hand and Jungian typology on the other. The organization of the data in electronic format as high-quality text scans identified only by unique code numbers ensures the double-blind nature of the study. As described earlier, every effort was made to avoid content and anchoring biases that could confound the judgment of the prospective handwriting analysts. The scanned handwriting samples, each with a code number, were combined and copied to a USB flash drive which was then mailed to each handwriting analyst. The flash drive also included a blank form the analyst would use to report the MBTI types 
for the 57 samples. Two additional questionnaires were included to obtain demographic information from each analyst (including the analyst's MBTI type), as well as soliciting any information about the analyst's method for typing the handwriting sample. When each analyst's reports were received, the unopened questionnaires were given to a graduate research student, who compiled the data.

\section{Results}

The four handwriting analysts determined MBTI code type using these 57 writing samples. The mean accuracy for identifying MBTI code type across all four handwriting analysts was $4.8 \%(S D=3.6 \%$, range $=$ $0 \%-5.3 \%)$. The accuracy across analysts did not significantly differ from chance, $t(3)=-0.78, p=0.76$. The mean accuracy across analysts for the individual dichotomies was $50.1 \%(S D=3.7 \%)$, which did not differ significantly from chance, $t(3)=0.06, p=0.96$. For the individual dichotomies, the mean accuracy across analysts was as follows: Extravert-Introvert $(\mathrm{EI})=46.5 \%(S D=3.0 \%)$, Sensation-Intuition $(\mathrm{SN})=51.7 \%(S D$ $=1.5 \%)$, Thinking-Feeling $(\mathrm{TF})=53.1 \%(S D=6.0 \%)$, and Judging-Perception $(\mathrm{JP})=49.1 \%(S D=5.0 \%)$. The analysts were not significantly better than chance when predicting any of the individual dichotomies (all $p$ 's $>$ $0.19)$.

Based on logistic regression models, the analysts were unable to significantly predict the individual dichotomy scores. The four analysts were not able to predict EI score, $X^{2}(4, N=53)=1.57, p=0.81$, NS score, $X^{2}(4, N=57)=4.15, p=0.39$, TF score, $X^{2}(4, N=57)=3.12, p=0.54$, or JP score, $X^{2}(4, N=57)=1.61, p=$ 0.81 . Individually, none of the analysts were able to significantly predict any of the individual dichotomies (all Bonferroni corrected $p$-values were $>0.3$ ).

In order to determine whether the four analysts rated the handwriting samples consistently (regardless of accuracy), Cohen's $K$ was used to measure inter-analyst agreement. The outcomes were as follows: EI $K=0.33$, SN $K=0.03$, TF $K=0.11$, JP $K=0.05$, with the overall four-letter MBTI code type $K=0.14$. All five $K$-values represent poor inter-analyst agreement, suggesting inconsistent ratings between the analysts.

We did some additional post-hoc analyses. Accuracy ratings of MBTI types based on these handwriting samples made by the study investigators before un-blinding did not differ from those of the handwriting experts. However, before un-blinding the investigators could discern gender based on handwriting significantly better than chance, supporting previous research (e.g., Beech \& Mackintosh, 2005; Bradley, 2015; Liwicki et al., 2007).

\section{Discussion}

Our findings do not permit us to refute the null hypothesis that handwriting experts are not able accurately to interpret the psychological types of individuals based on these individuals' handwriting samples. Although the use of a double-blind cohort research design and the use of a standardized text that did not provide any content cues about a participant constitute improvements in methodology over previous studies, there are still some limitations of the present study that should be acknowledged. These limitations lie in four domains: the adequacy of the criterion of known psychological type, the representativeness of the cohort, the "black box" nature of the handwriting analytic method used, and the representativeness and qualifications of the handwriting analysts. First, although we assumed that the self-typing of expert long time users and teachers of psychological type was a reasonable strategy, this criterion was not verified through independent ratings by instruments or reports of collateral informants. Second, the distribution of the 16 Types in this cohort sample 
was overrepresented with professionals reporting an intuitive preference. A better procedure would have been to have selected a cohort of participants whose MBTI preferences were representative of the frequencies of each MBTI code type in the general population. However, the strong inference about being able to derive a MBTI type from a handwriting sample should not depend upon the analyst rater's knowledge of the base rate of that type in the cohort sample, and we have no indication that our four analysts applied such a presumption about base rates in making their type determinations. Third, the handwriting analysts used their own methods for deriving MBTI code types from the handwriting samples, i.e., it was a "black box" process. A consensus protocol for interpreting Jungian types would be desirable, but such a consensus protocol for Jungian typing of handwriting does not exist to our knowledge. Finally, it is likewise possible that the handwriting analysts that we recruited were not representative of analysts who are most knowledgeable about interpreting MBTI types from a standardized handwriting sample. Having a wider diversity of handwriting analysts of different psychological types who also were trained to a specified level in terms of their knowledge of Jungian typology and the MBTI would be a design improvement.

\section{Conclusions}

Although these findings do not allow us to refute the null hypothesis that Jungian Typology (as indicated by the MBTI) cannot be inferred from de-identified cursive handwriting samples obtained from copying standardized texts, we have identified some improvements for this research project. Our next steps include two strategies: (1) Develop consensus Jungian type handwriting coding criteria, train new raters, and repeat this study with blind ratings using these new criteria; (2) Using data from a large undergraduate sample, develop reliable criteria for coding handwriting features, and then determine if any pattern of these features differentiate the 16 Types and/or dichotomies on the function and attitude pairs.

\section{References}

Bayne, R., \& F. O’Neill (1988). Handwriting and personality: A test of some expert graphologists' judgments. Guidance \& Assessment Review, 4, (4), 1-3.

Beech, J. R., \& Mackintosh, I. C. (2005). Do differences in sex hormones affect handwriting style? Evidence from digit ration and sex role identity as determinants of the sex of handwriting. Personality and Individual Differences, 39, 459-468.

Beyerstein, B. L. (1992). The origins of graphology in sympathetic magic. In B. L. Beyerstein, \& D. F. Beyerstein (Eds.), The write stuff: Evaluations of graphology-The study of handwriting analysis (pp. 163-200). Buffalo, N.Y.: Prometheus.

Beyerstein, B. L., \& Beyerstein, D. F. (1992). The write stuff: Evaluations of graphology-The study of handwriting analysis. Buffalo, N.Y.: Prometheus.

Bradley, S. (2015). Handwriting and gender: A multi-use data set. Journal of Statistics Education, 21(1), 1-15.

Center for Applications of Psychological Type (CAPT). (1985). An appreciation of Isabel Briggs Myers (special commemorative issue). MBTI News, 2(4).

Gray, H., Wheelwright, J. H., \& Wheelwright, J. B. (1964). Jungian Type Survey. San Francisco, C.A.: Jung Institute.

International Graphoanalysis Society. (IGAS). (2010). Our company. Retrieved May 4, 2010, from http://graphoanalysis.com/ company.asp

Jung, C. G. (1923). Psychological types (Trans. H. G. Baynes; rev. ed., 1971, Trans. R. F. C. Hull). Princeton, N.J.: Princeton University Press.

Jung, C. G. (1933). A psychological theory of types. In Modern man in search of a soul (Trans. W. S. Dell \& C. F. Baynes, pp. 74-94). San Diego, C.A.: Harcourt Brace.

Kahneman, D., \& A. Tversky (1973). On the psychology of prediction. Reprinted in D. Kahneman, P. Slovic, \& A. Tversky (1982), Judgment under uncertainty: Heuristics and biases (pp. 48-68). New York: Cambridge University Press.

Keirsey, D., \& M. Bates (1984). Please understand me: Character and temperament types. Del Mar, C.A.: Prometheus Nemesis. 
Kopischke, T. L. (2001). Content analysis, graphology, and personality assessment: A cross-validation (Doctoral dissertation, University of Virginia).

Lawrence, G. (1986). Issues in the development of the MBTI. In Home study notebook, MBTI training program (pp. 2.11-2.23). Gainesville, F.L.: Association for Psychological Type.

Lester, D., \& R. J. Hoyd (1981). Handwriting slant and thinking/feeling. Perceptual and Motor Skills, 53, 258.

Liwicki, M., Schlapbach, A., Loretam, P., \& Bunke, H. (2007). Automatic detection of and handedness from on-line handwriting. Proceedings of the 13th Conference of the International Graphonomics Society, pp. 179-183.

Lockowandt, O. (1976). The present status of research on handwriting psychology as a diagnostic method. Reprinted in B. L. Beyerstein \& D. F. Beyerstein (1992), The write stuff: Evaluations of graphology-The study of handwriting analysis (pp. 55-85). Buffalo, N.Y.: Prometheus.

Majors, M. S. (2012). Majors personality type elements users manual. San Jose, C.A.: Acer Press.

Moore, M. (1982). Jungian psychology: Graphological applications. Charlottesville, V.A.:Author.

Myers, I. B. (1980). Gifts differing. Palo Alto, C.A.: Consulting Psychologists Press.

Nickell, J. (1992). A brief history of graphology. In B. L. Beyerstein, \& D. F. Beyerstein, The write stuff: Evaluations of graphology-The study of handwriting analysis (pp. 23-29). Buffalo, N.Y.: Prometheus.

Oskamp, S. (1965). Overconfidence in case study judgments. Reprinted in D. Kahneman, P. Slovic, \& A. Tversky (1982), Judgment under uncertainty: Heuristics and biases (pp. 287-293). New York: Cambridge University Press.

Sesa-Nogueras, E., Faundez-Zanuy, M., \& Roure-Alcobe, J. (2015). Gender classification by means of online uppercase handwriting: A text-dependent allographic approach. Cognitive Computation, 7(2), 1-15. DOI 10.1007/s12559-015-9332-1.

Sevilla, M. (1996). Relationships between handwriting analysis and the Myers-Briggs Type Indicator in assessing personality (Doctoral dissertation, California Graduate Institute).

Singer, J. K., M. Loomis, E. Kirkhart, \& L. Kirkhart (1996). The Singer-Loomis Type Inventory. Gresham, O.R.: Moving Boundaries.

Teillard, A. (1973). L' ame et l'ecriture. Paris: Villain \& Belhomme. (in French) 